\title{
Effect of vitamin D supplementation on inflammation and nuclear factor kappa-B activity in overweight or obese adults
}

\author{
Aya Mousa ${ }^{1}$, Negar Naderpoor ${ }^{1}$, Josphin Johnson ${ }^{1}$, Karly Sourris ${ }^{2}$, Kirsty Wilson ${ }^{3}$, Magdalena
}

Plebanski $^{3}$, Maximilian PJ de Courten ${ }^{4}$, Barbora de Courten ${ }^{1}$

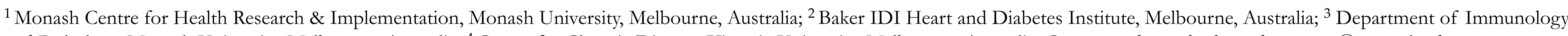
and Pathology, Monash University, Melbourne, Australia; ${ }^{4}$ Centre for Chronic Disease, Victoria University, Melbourne, Australia. Correspondence: barbora.decourten@monash.edu

\section{Background}

\section{- Chronic low-grade inflammation is} common in obesity and its related conditions, including type 2 diabetes.

- Experimental studies have shown that vitamin $\mathrm{D}$ regulates inflammation via inhibiting the nuclear factor-kappa B $(\mathrm{NF} \kappa \mathrm{B})$ pathway; however clinical trials to support these findings are lacking.

\section{$\underline{\text { Aims }}$}

To examine the effect of vitamin D supplementation $(100,000$ IU bolus + 4,000 IU daily cholecalciferol for 16 weeks) on inflammation markers and $\mathrm{NF}_{\kappa \mathrm{B}}$ activity, compared to placebo, in vitamin $\mathrm{D}$-deficient and overweight or obese adults.

\section{Hypothesis}

We hypothesized that vitamin D supplementation would reduce inflammation markers and $\mathrm{NF \kappa B}$ activity, compared to placebo.

\section{Inclusion Criteria:}

Methods

- healthy, non-diabetic adults (WHO guidelines); age 18-60 years with body mass index $(\mathrm{BMI}) \geq 25 \mathrm{~kg} / \mathrm{m}^{2}$.

- vitamin D deficient: 25 -

hydroxyvitamin $\mathrm{D}(25 \mathrm{OH}) \mathrm{D}) \leq 50$ $\mathrm{nmol} / \mathrm{L}$

- non-smokers; no heavy alcohol consumption, not using illicit drugs, medications, vitamins, supplements

- no clinical or laboratory signs of acute or chronic inflammation

- no hypercalcemia, allergies, cancer in last 5 years, menopause, pregnancy, or lactation

\section{Intervention/Control:}

- double-blind random allocation to 100,000 IU single bolus + 4,000 IU daily oral cholecalciferol or identical placebo capsules for 16 weeks

\section{Outcome measures:}

- anthropometry: BMI, waist-to-hip ratio (WHR), and \% body fat by dual X-ray absorptiometry (DEXA)

- serum 25(OH)D: chemiluminescent assay

- high-sensitivity C-reactive protein (hsCRP): rate turbidimetry

- tumor necrosis factor (TNF), monocyte chemoattractant protein-1 (MCP-1) interleukins (IL)-1B, 6, 8, 10, 12, 18, 23 and 33: multiplex assay; flow cytometry

- NFкB activity in peripheral blood mononuclear cells: DNA-binding assay

\section{Ethics \& Registration:}

- Monash University Ethics (CF13/ 3874-2013001988); clinical trials.gov (ID: NCT02112721).

Fig 2. Change in serum 25(OH)D was not associated with change in NFKB activity

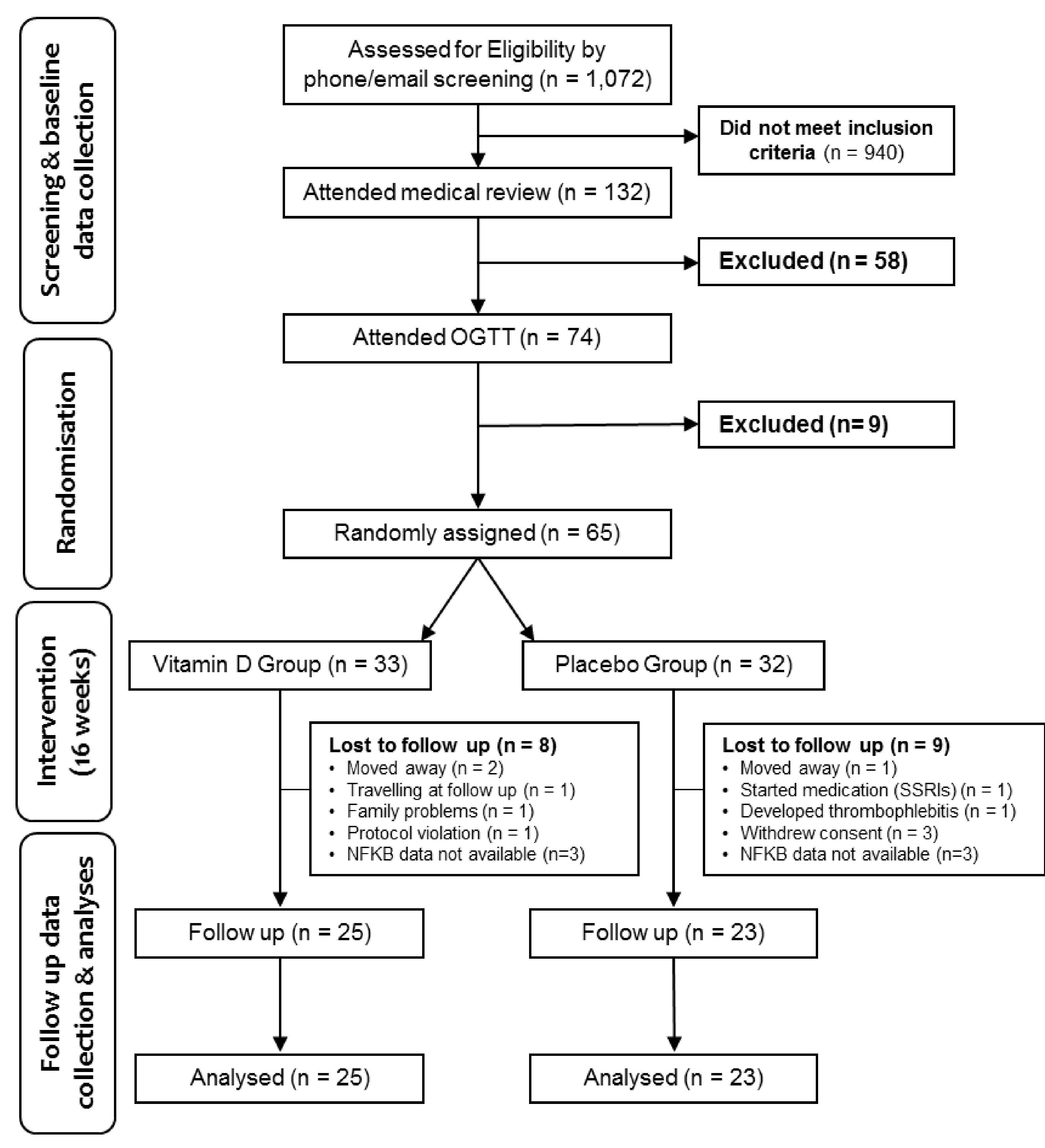

- Forty-eight participants completed the study (30M/19F; age $=31.7 \pm 8.5$ years). Age and sex did not differ at baseline $(p>0.3)$. Other characteristics are shown in Table 1.

Fig 1. Serum 25(OH)D levels increased significantly in the vitamin D group

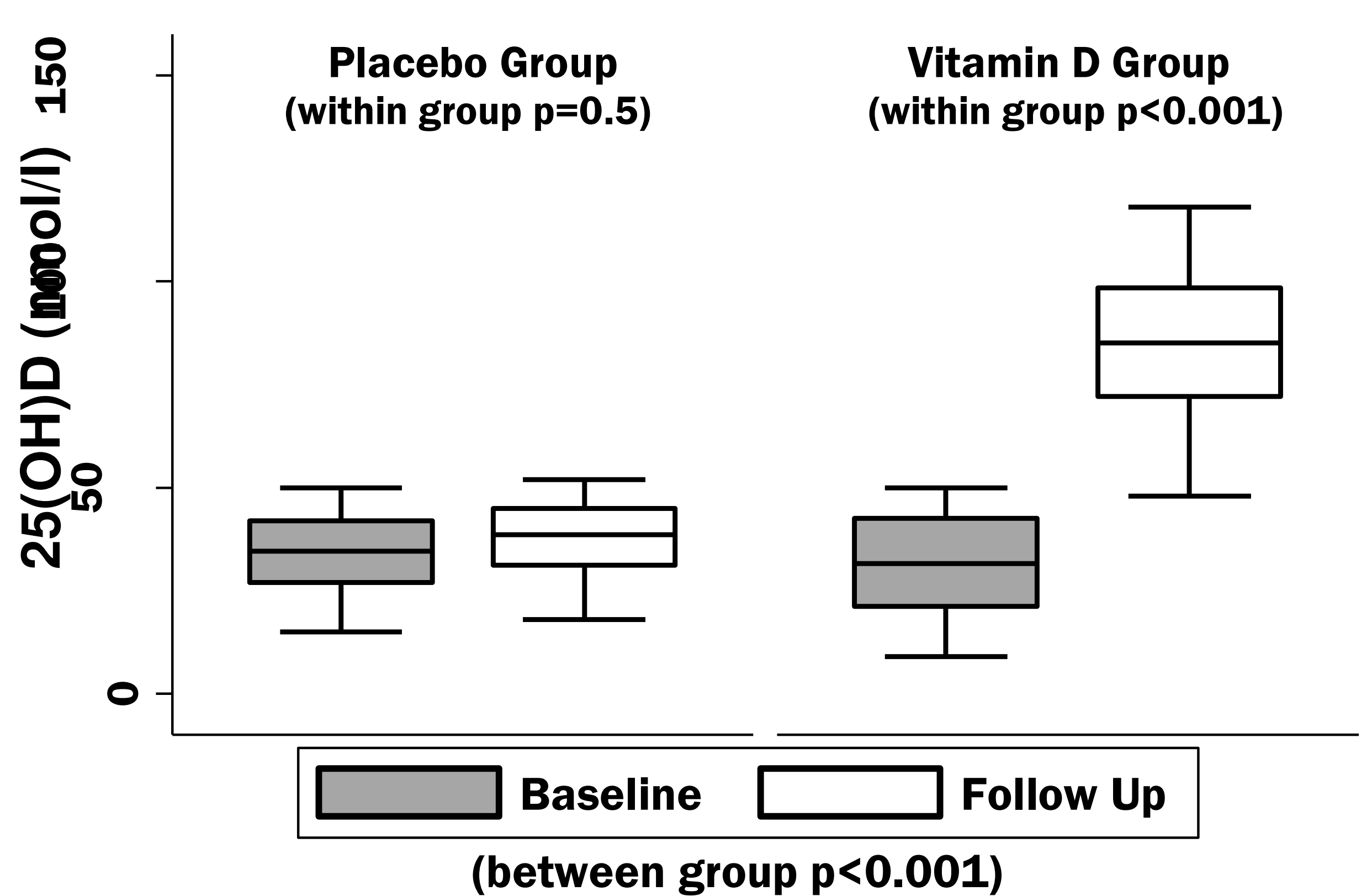

- Serum 25-hydroxyvitamin D levels increased in the vitamin D group from 31.4 to $88.8 \mathrm{nmol} / \mathrm{L}$ ( $\mathrm{p}<0.001$ ), with no change in the placebo group (35.0 to 33.4 , $\mathrm{p}=0.5$ ) (Fig 1; Table 1).

- Change in $25(\mathrm{OH}) \mathrm{D}$ was not associated with change in $\mathrm{NF} \kappa \mathrm{B}$ activity (Fig 2) or inflammatory markers before or after adjustment for age, sex and \% body fat (all $p>0.05$ ).

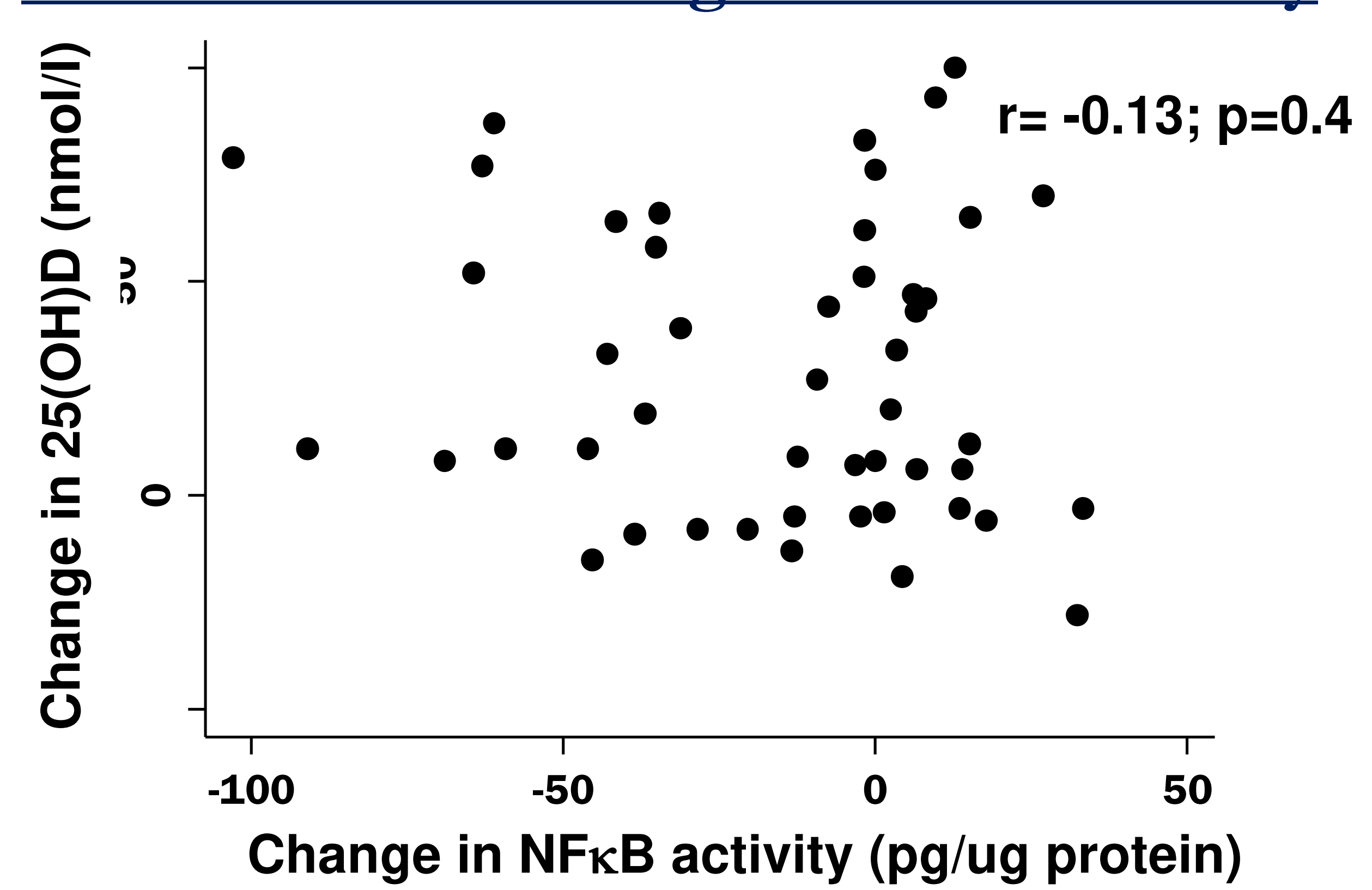

Fig 3. No difference in NFKB activity between vitamin $\mathrm{D}$ and placebo groups

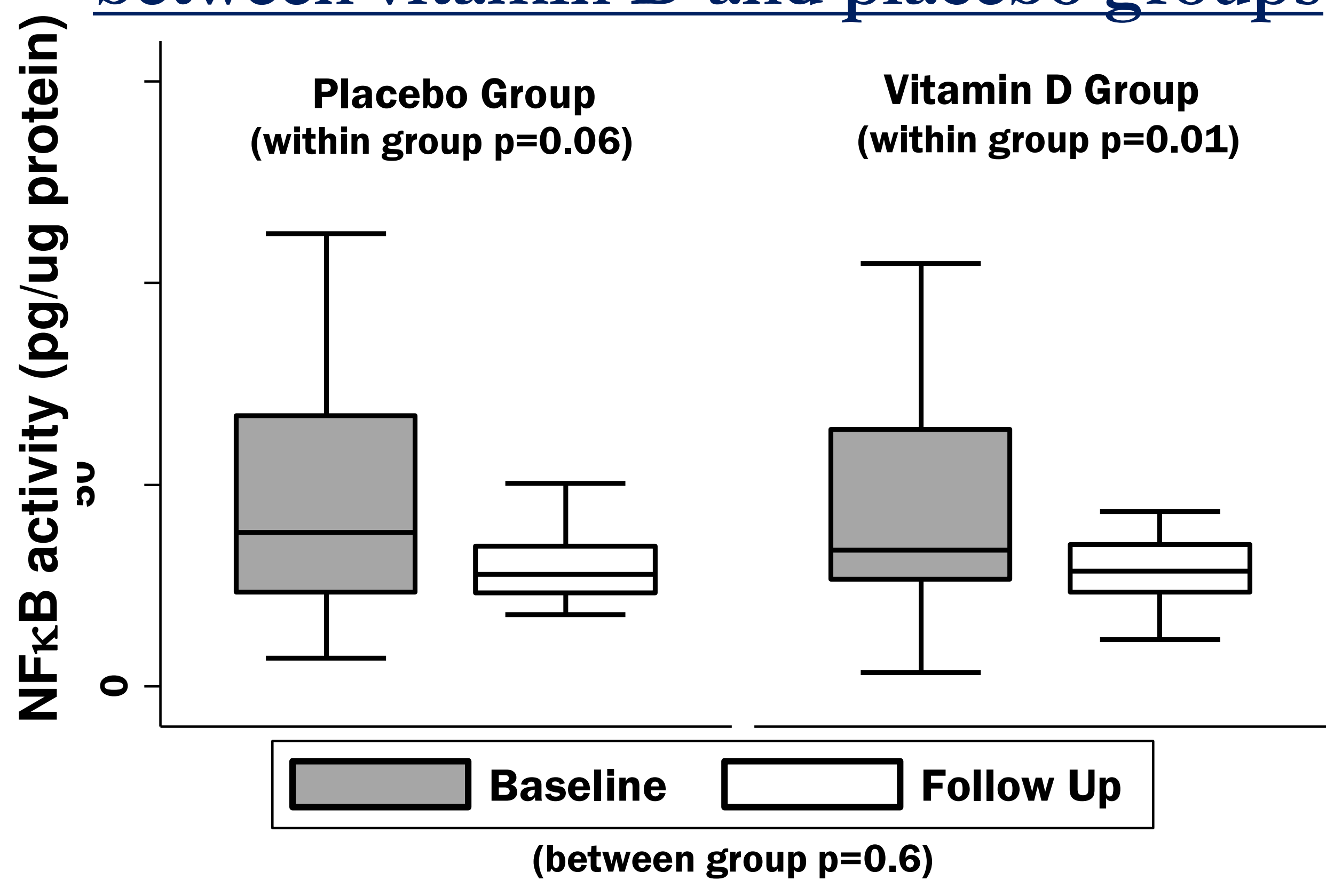

- $\mathrm{NF}_{\kappa} \mathrm{B}$ activity decreased significantly within the vitamin $\mathrm{D}$ group (with a non-significant trend within the placebo group); however there was no difference in change in $\mathrm{NF \kappa B}$ between groups ( $p=0.6$; Fig 3 ).

- There were no differences between groups in change in any inflammation markers (all p>0.05).

- All results remained non-significant after adjustment for baseline values, age, sex and \% body fat, and after further adjustment for dietary vitamin D intake, sun exposure and physical activity.

\section{Conclusions}

- Although experimental studies show that vitamin D reduces inflammation by inhibiting NFKB activity, data from our RCT shows no effect of vitamin D supplementation on inflammation markers or $\mathrm{NF \kappa B}$ activity in vitamin $\mathrm{D}$-deficient and overweight or obese, otherwise healthy adults.

- Larger trials are needed to confirm these findings, particularly in participants with diseases associated with low-grade inflammation, such as type 2 diabetes.

\section{$\underline{\text { References }}$}

Yu et al.(1995) Immunology, 92: 10990-994. - Lundqvist et al. (2014). Steroids, 85: 30-35.

\begin{tabular}{|c|c|c|c|c|c|c|c|c|c|c|}
\hline \multirow{2}{*}{ Variable } & \multicolumn{4}{|c|}{ Vitamin D group $(n=25)$} & \multicolumn{4}{|c|}{ Placebo group $(n=23)$} & \multirow[b]{2}{*}{$P_{3}$} & \multirow[b]{2}{*}{$P_{4}$} \\
\hline & Baseline & Follow Up & $P_{1}$ & Change & Baseline & Follow Up & $P_{2}$ & Change & & \\
\hline BMI $\left(\mathrm{kg} / \mathrm{m}^{2}\right)$ & $31.8 \pm 4.9$ & $31.9 \pm 4.9$ & 0.8 & $0.04 \pm 0.9$ & $30.7 \pm 3.9$ & $30.6 \pm 4.5$ & 0.7 & $-0.1 \pm 1.2$ & 0.4 & 0.6 \\
\hline Body fat (\%) & $41.4 \pm 8.0$ & $40.9 \pm 8.0$ & 0.1 & $-0.5 \pm 1.5$ & $40.2 \pm 9.0$ & $39.7 \pm 9.0$ & 0.2 & $-0.5 \pm 1.7$ & 0.6 & 0.9 \\
\hline $\begin{array}{l}25(\mathrm{OH}) \mathrm{D} \\
(\mathrm{nmol} / \mathrm{l})\end{array}$ & $31.4 \pm 12.5$ & $88.8 \pm 21.5$ & $<0.001$ & $57.4 \pm 22.5$ & $35.0 \pm 10.4$ & $33.4 \pm 13.4$ & 0.5 & $-1.6 \pm 10.9$ & 0.3 & $<0.001$ \\
\hline $\begin{array}{l}\mathrm{NF} \kappa \mathrm{B}(\mathrm{pg} / \mu \mathrm{g} \\
\text { protein) }\end{array}$ & $47.7 \pm 30.2$ & $29.9 \pm 12.5$ & 0.01 & $-17.8 \pm 31.9$ & $45.6 \pm 29.5$ & $32.4 \pm 18.7$ & 0.06 & $-13.2 \pm 32.4$ & 0.8 & 0.6 \\
\hline
\end{tabular}

$P$-values represent $t$-tests for differences within vitamin D group $\left(P_{1}\right)$ or placebo group $\left(P_{2}\right)$, or $t$-tests for differences at baseline $\left(P_{3}\right)$ or differences in change scores $\left(P_{4}\right)$ between groups. 